Future directions in personality pathology

\title{
Future directions in personality pathology
}

\begin{abstract}
This article suggests two areas of future development in the realm of personality pathology. Firstly, a reconceptualization of personality pathology in the context of research evidence suggestive of a single model for psychopathology. Recent work on the "p factor", in combination with findings from clinical research, behavior genetics, molecular biology and neurobiological models are considered in relation to this reconceptualization of personality pathology. Secondly, a cultural-developmental model for personality pathology is proposed, based on the Gergely and Csibra's work on natural pedagogy, Tomasello's work on joint attention and intentionality, and our recent work on epistemic trust and the socialcommunicative nature of psychopathology.
\end{abstract}

Key words: personality disorder, mentalization, epistemic trust

\section{Introduction}

We will discuss here two main areas of potentially fruitful future development in understanding personality pathology and how they might be linked to provide a more integrated framework for thinking about personality disorder. The first area involves the conceptualization of mental disorder. The second area we would like to explore is a new cultural-developmental model for psychopathology that more fully integrates the role of the social environment into developmental psychopathology. 
Future directions in personality pathology

\section{A singular model of mental disorder}

In thinking about how we categorise mental disorder, and the role of personality disorder more particularly within that, we suggest that a model of psychopathology that moves away from specific categorical diagnoses and focuses on what may be common to different types of mental disorders may be more helpful in capturing the dysfunctions underpinning personality disorder. We believe that this approach might more accurately depict the clinical, phenotypical and biological reality of personality disorder, rather than excessive reliance on the intricate taxonomic system traditionally used [1].

The evidence base in support of a general model derives from different research domains. The first relates to the clinical difficulties arising from the high frequency of comorbidity across psychiatric disorders in general $[2,3]$. Comorbidity cuts across diagnoses and is observed between most symptoms (e.g. [4]). It is notably prevalent in personality pathology in particular $\left[5,6^{*}\right]$. If disorders were understood as indicators of latent transdiagnostic spectra, comorbidity might more meaningfully be substituted with the concept "frequent co-occurrence" [7]. Inconsistent conceptualization and assessment of severity is also a clinical problem. Zimmerman and colleagues indicate that we lack agreed concepts of severity, both across diagnostic categories and even within disorders [8*]. Instead, severity is gauged unevenly in terms of the number, intensity, frequency or persistence of symptoms, quality of life, and risk of disability or mortality Does severity overlap with what is currently frequently attributed to 'personality pathology'? By some definitions they appear to be loosely coupled constructs, particularly in terms of temporal course and the persistence of acute distress.

The second area of work relevant to the unified model is the emerging literature on bifactor modelling of symptoms and diagnoses. Such models assume that all symptoms (or diagnoses) are in part determined by a common source of variance - a single factor - and the 
Future directions in personality pathology

residual variance may then cluster into factors that resemble diagnostic categories of personality disorders or groupings of diagnostic groups (spectral-level factors). Work in this area over the past decade by Lahey, Caspi, and others points to the existence of a general psychopathology factor - labelled the p factor - which was assumed to exist alongside clusters of symptoms grouping into internalizing, externalizing, and psychosis factors $\left[9^{* *}, 10\right]$. Studies across numerous samples in children, adolescents, and adults $[11,12 * *, 13]$ suggest that the $\mathrm{p}$ factor appears to capture an underlying propensity for any kind of psychopathology, captured as a global statistic, which is in itself then open to external validation. A key study by Sharp and colleagues applied the $\mathrm{p}$ factor to understanding borderline personality disorder (BPD). In this study, with a sample of 966 inpatients, bifactor analysis revealed that once the common symptoms across multiple personality disorder diagnoses were accounted for in this way, BPD was best understood as coterminous with the general factor that underpins an individual's level of vulnerability to personality disorder symptoms of all kinds, rather than as a separate diagnosis [14*]. In other words, BPD symptomatology might be understood as capturing a core common to all other expressions of personality disorder. It has further been suggested that BPD might be seen as an expression of an individual's position on the $\mathrm{p}$ factor spectrum, associated with greater risk, higher levels of comorbidity, and increased symptom severity [15].

Examining classic questions, such as whether personality disorder disrupts the likelihood of successful psychiatric or psychological treatment, may be best studied using bifactor analysis. Perhaps the explanation of conflicting results in this area lies in the use of diagnoses of individual personality disorders as moderators of treatment outcome rather than a global construct of personality dysfunction represented by a general latent factor which predicts all indicators of personality disorder. We should note, however, that there have been some important critiques of the $\mathrm{p}$ factor as a statistical construct. One such position argues 
Future directions in personality pathology

that the fact that the same symptoms occur in disorders (for example, irritability are symptoms of both depression in adolescence and antisocial personality disorder) may generate simply the illusion of a general psychopathology factor. A study, however, that took out these common symptoms still observed the general model intact [16]. It has also been suggested that nuisance variables - for example, common method variance, shared biases, positive or negative halo effects - could explain a general factor. It has also been argued that the bi-factor model may appear superior because it more efficiently overfits the data, or it is the statistical complexity rather than any substantive properties that generates the appearance of a better fit. A further critique is that bi-factor model may only be functioning as a mechanism for the reallocation of covariance across indicators rather than actually being a novel model $[17,18]$. Rightly, there are calls for more work to be done to test both specific and general dimensions against substantive measures [19,20]. More work, therefore needs to be undertaken in this area both clarifying statistical questions and independently validating the $\mathrm{p}$ factor in order to exclude the potential of incorporating an artefact rooted in method being inappropriately incorporated in causal explanations.

Third, findings from behavior genetics and molecular biology are consistent with a common-factor argument. Studies of families and twins tend to show that genetic risk is not specific to particular disorders, but is largely a transdiagnostic vulnerability factor [21-24]. In terms of etiology, twin studies show that genetic and environmental effects on a general factor appear to be roughly equal [25,26]. In family studies, increased relatedness is associated with a general increase in risk of mental disorder, given a diagnosis of severe mental disorder in a proband (e.g. [27]). The notion of a general vulnerability to psychopathology, regardless of diagnosis, is supported by molecular genetic studies, which confirm that phenotypic similarity can be accounted for by concordance at the molecular level $[28,29]$. A genome-wide association study of 25 brain disorders and 17 mental disorders 
Future directions in personality pathology

from over 1 million participants revealed that mental disorders shared common variance risks [30]. This commonality was less evident for brain disorders - which are normally considered neurological - which appear more distinct from each other. Following such indications, perhaps we could contemplate a gentle move away from searching for specific causes and mechanisms linked with individual disorders before focusing on general vulnerability to disorder per se. Identifying common mechanisms of causation across diagnostic groups may help establish a new position for personality dysfunction in the etiology of mental disorder.

Dysfunction of the prefrontal cortex is consistently implicated in transdiagnostic neurobiological approaches to psychopathology [31*], with reduced grey matter volume in the prefrontal cortex and limbic regions of particular salience [32]. Emerging findings from neurobiological research suggest the presence of structural anomalies in grey matter in the cerebellum (which has a role in coordinating thought and affect and white matter integrity) $[33,34]$ or in the body of the corpus callosum [35,36*]. Neuro-behavioural processes that mediate the association between brain and psychological function across disorders and irregular cortical connectivity might be an endophenotype of general psychopathology [37]. In this spirit we could review common factors not just in pathology but also in the treatment of mental disorder, where specificity in terms of either diagnostic entities or treatment modalities has turned out to be a poor predictor of therapeutic outcome.

There is a significant body of research on the strong associations between psychopathology and emotion dysregulation [38*]. In general, brain structure, neural function, and behavioral evidence converge to suggest that transdiagnostic psychopathology in some part stems from emotion dysregulation [39]. This conclusion is greatly strengthened by the growing use of emotion-regulation-inspired interventions to treat various kinds of mental disorder [40-43]. In particular, third-wave psychological interventions such as Dialectical Behavior Therapy (DBT) [44], Acceptance and Commitment Therapy [45], 
Future directions in personality pathology

mindfulness-based interventions [46] and the Unified Protocol for Emotional Disorders [47], directly target emotion regulation as a component of treatment $[48,49]$.

\section{A cultural-developmental model of psychopathology}

Focusing solely on the biological roots of personality dysfunction excludes centuries of accumulated wisdom concerning the social determinants of mental disorder. The search for a likely unifying cause may also bring into a common framework the well-known key risk factors associated with individual (e.g., ethnicity), socioeconomic (e.g., low household income), family history (e.g., adverse childhood experiences, poor parental mental health), and the wider social environment (e.g., homelessness, criminal involvement). If there were a common psychosocial cause, a core to personality dysfunction, it would have to be something fairly core to the emergence of the human species. One key universal is the culture-making capacity of the human mind, bringing with it a sensitivity to social learning and a complex social imagination. We suggest that this cultural model might provide some corrective to developmental approaches to mental disorder that have inadequately acknowledged the role of the wider social environment in creating risk or resilience in relation to mental health disorder.

This thinking originates in Csibra and Gergely's theory of natural pedagogy [50], and the work of Tomasello and colleagues [51,52] on the role of joint attention in human cognition and the transmission of culture. Csibra and Gergely have described natural pedagogy as a social cognitive adaptation dedicated to ensuring the most effective and efficient transfer of culturally relevant knowledge. This adaptation allows humans to transmit cultural knowledge - that is, information that is generalizable and regarded as shared, in that the expectation is that others belonging to a given social group also possess the same knowledge. Natural pedagogy is highly dependent on and sensitive to ostensive cues. 
Future directions in personality pathology

Originally described by Bertrand Russell [53] and developed by Sperber and Wilson [54], ostensive cues signal the intention of one person to communicate knowledge to another [50].

Csibra and Gergely found that infants were particularly sensitive to certain behavioral signals that alerted the infant to the relevance of a communication aimed at them (e.g., establishing eye contact, eyebrow raising, turn-taking contingency, being addressed in "motherese" or unfamiliar words [50,55,56]). Infants recognize communicative and informative intentions in the cues the informant uses $\left[57^{*}, 58\right]$. Having recognized these cues, the recipient of a communication can relax their epistemic vigilance, described by Sperber and Wilson [54] as a necessary protector against being misinformed. Once trust in a communicator (and in the information they seek to communicate) has been established, humans are open to internalize and assimilate the knowledge offered by the communicator, in order to take in the complex and sophisticated cultural knowledge required to navigate the social environment [59-61]. This work also builds on Vygotsky's account of how children learn within interpersonal relationships that scaffold learning and provide opportunities for practice. Through internalization and representation, these skills bed down as established social and cultural knowledge [62].

Throughout the course of life, humans are highly responsive to ostensive cues that stimulate epistemic trust. Any action that involves a communicator recognizing a listener as being agentive acquires this ostensive quality, and potentially primes the listener to adopt an attentional state to subsequent communications. The learning system envisioned by Csibra and Gergely [50] for the transmission of cultural knowledge is triggered by the experience of mutual recognition of intent. Mentalizing (or reflective functioning), which involves the recognition of the other's agency and interest in their mind, operates as a form of ostensive signalling, establishing a shared intentional structure in learner-directed actions and triggering the learning system that Gergely and Csibra describe. We have argued that 
Future directions in personality pathology

mentalizing is important because it is such a powerful cue for the opening of epistemic trust that is needed for individuals to benefit fully from opportunities to learn from others about the world in general and their social world in particular [15,63-65]. The key to keeping the learning channel open is the experience of self-recognition that enables genuine learning from the communicator This self-recognition occurs through identifying how one is seen by the other, and appreciating that the other person's perception of oneself is congruent with one's own. This congruence creates what we have called an "epistemic match" and it leads to opening of the channel for efficient social communication - making social learning, affect regulation, and flexible and adaptive social functioning more possible.

This thinking emphasizes the highly interpersonal nature of the process by which epistemic trust is created and sustained. In terms of developmental psychopathology, one implication is that if an infant has caregivers who are not reliably responsive or able to recognize what is meaningful and relevant to the infant, this can undermine the infant's development of epistemic trust. Early adversity, severe neglect, or maltreatment - perhaps the most generally agreed transdiagnostic cause of mental disorder - may arguably be the most damaging way in which social learning is disrupted. Such experiences involve exposure to an extreme "epistemic mismatch", or even epistemic conflict, in which a child's knowledge of the world - the physical and emotional data of their own experience - is not only unrecognized by the caregivers but also actively denied, repudiated, or punished. In these circumstances, a child might close down their instinct for social learning as an adaptation to a social environment that cannot be trusted as a reliable source of information.

Two conclusions would follow from this line of argument. First, we would anticipate that adversity, or more specifically relational trauma such as severe neglect or abuse, would generate problems of adaptation in a learning context. We suggest that personality pathology might be more accurately understood as reflecting the breakdown in social communication 
Future directions in personality pathology

that arises from an entrenched breakdown in epistemic trust. Specifically, we would predict that the mechanism of learning about oneself from others is disrupted following a traumatic experience. Therefore, we would anticipate that the capacity for mentalizing would be limited by a history of adverse interpersonal experiences, at least in some individuals. Second, we would anticipate an inevitable repetition of traumatic experience in the children of individuals who have been traumatized.

Evidence is accumulating that the capacity for orienting effectively to the mental state of others is dramatically reduced following adversity. For example, it is well known that children with a history of early life stress, particularly neglect or abuse, struggle to recognize emotional facial expressions, and the degree of their inaccuracy predicts their general social competence $[66,67]$. Adolescent girls with a diagnosis of BPD have been shown to need more perceptual information than those without such a diagnosis in order to accurately judge simple facial expressions of happiness and anger [68]. We have recently systematically reviewed the literature on the impact of trauma on the development of social cognition [69**]. In particular, complex trauma (i.e., early negative life experiences involving neglect and/or abuse, typically within an attachment/caregiving context) can lead to mentalizing impairments, often in the form of strongly biased mentalizing, hypersensitivity to the mental states of others, a defensive inhibition of mentalizing, or a combination of these features (for reviews, see [70,71]).

Until now, we have considered mentalizing impairments as an indication of the impact of trauma on cognitive functioning [72-74]. It may more accurately be seen as an adaptation to the social environment. In such circumstances it may be a better strategy to regard communications from others as irrelevant, suspect, or misleading. The problem with such a strategy is that in the longer term, the individual is unable to reap the full benefits of social learning. Access to other qualities of the social environment that normally support 
Future directions in personality pathology

psychological resilience in the face of challenges - such as being able to seek and receive help, having a social network, or being open to change - is potentially lost. We have emphasized the role of early adversity here, but for anyone, operating in a nonmentalizing system (whether the family environment or a hostile social environment or institution) takes a toll on psychological resilience because it precludes the kind of epistemic congruence that underpins the protective factors of adaptive flexibility and a regulated sense of self and of self-in-relation-to-others. This approach suggests that disruptions in epistemic trust are central features of most severe psychopathologies and argues that re-establishing epistemic trust may be the core common factor behind most effective psychological therapies, allowing the existence of a therapeutic alliance and subsequently facilitating the re-emergence of social learning $[15,63,65]$.

Beyond adversity, many other cultural influences on personality function could be mediated by systemic epistemic mistrust. Seeing personality functioning as overlapping with social connectedness is not a new idea, but linking in the mechanism of social learning and social communication offers a new angle on this issue. From this perspective, personality disorder is less a disorder than an adaptation gone awry through its entrenchment, which is mistaken for a characteristic of personality as it entails a certain rigidity - that is, a failure to respond appropriately to changing social circumstances. The attribution to character may be an understandable misattribution. At the core of personality dysfunction is a failure of trust in new knowledge that is conveyed in social communication. Social conditions associated with personality dysfunction may impact on an individual by creating a tendency for epistemic hypervigilance and distrust, closing internal channels of social learning and social connection, leaving the individual vulnerable to the pressures and strains of the social environment. This line of thinking has been actively elaborated by social network theorists advancing a set of concepts that elaborate specific features about how individuals are 
Future directions in personality pathology

connected $[75,76]$. This is a relatively recent domain within quantitative social science, which has generated impressive mathematical models of social influence. There is accumulating evidence that mental health (e.g. suicidal ideation [77]), wellbeing (e.g. alcohol consumption [78]), and aspects of function pertinent to mental health (e.g. being a victim of violence) are strongly influenced by an individual's position and proximity of contact with or separation from effective social influence. Mathematical models of social networks can guide interventions [79] and address developmental vulnerabilities or openness to social influence. The potential reach, as well as feasibility and likely acceptability, of interventions motivated by social network theory may be a fruitful avenue in the study of personality function.

\section{Conclusion}

The developmental, dimensional approach that we have described here as a possible future direction for thinking about personality pathology leaves us with immediate suggestions about the treatment of PD. In particular, our emphasis on the developmental origins of PD and the role of the social environment in supporting resilience or increasing risk suggests that we need to think more actively about engaging with the treatment and management of personality pathology in adolescents. There has traditionally been some (understandable) concern about the dangers of labelling and stigma in relation to diagnosing PD in adolescents. We suggest however, that these worries perhaps reflect clinicians' anxieties and perceptions around the concept of PD, rather than those of the young people themselves, who are in most cases more concerned with finding the help and support they require and deserve. The work of the Global Alliance for the Prevention and Early Intervention for BPD (GAP) is an example of a programme to encourage early detection and prompt intervention for personality pathology. Working with families to provide ongoing support and with different agencies to develop knowledge and understanding, GAP provides a step on a path leading us 
Future directions in personality pathology

in the right future direction to create a social environment - at least clinically - that can best support vulnerable young people.

The issues that discussion of BPD in adolescence provokes around finding the appropriate balance between concerns about diagnosis and its implications and the need for timely support and intervention brings us back to the $\mathrm{p}$ factor and the value of approaching it from a developmental perspective. Some of the hesitation around diagnosing BPD or even $\mathrm{PD}$ in adolescence arises from the frightening and reifying prospect it presents for an individual and their families to consider. Those with BPD, particularly in its more severe forms, face encountering high levels of distress and the need for high levels of support and treatment. We would like to emphasise that by thinking of such a diagnosis within the context of the $\mathrm{p}$ factor idea allows us to integrate facing the difficult reality of severe symptomology alongside a capacity to anticipate ebb and flow in relation to disorder. An individual may well have the same underpinning $\mathrm{p}$ factor vulnerability - as an outcome of a confluence of genetic vulnerability and developmental experience - but the playing out of that vulnerability across the life course is highly variable and contingent on external stressors and, critically, the presence or absence of support at particular stages. Individuals with PD have traditionally been regarded as particularly difficult to help; we now know that there are effective psychosocial treatments. We suggested above that vulnerability to pathology is an outcome of human cultural-cognitive capacities; the converse of this is such capacities have also given us the means to provide social scaffolding to our conspecifics. It may be the provision of that social scaffolding that the therapeutic armamentarium of PD most urgently needs.

\section{Funding}


Future directions in personality pathology

Peter Fonagy is in part supported by the NIHR Applied Research Collaboration (ARC) North Thames at Barts Health NHS Trust. The views expressed are those of the author(s) and not necessarily those of the NIHR or the Department of Health and Social Care.

\section{References}

1. American Psychiatric Association: Diagnostic and statistical manual of mental disorders, 5th edn edn 5th. Washington, DC: American Psychiatric Association; 2013.

2. Kessler RC, Chiu WT, Demler O, Merikangas KR, Walters EE: Prevalence, severity, and comorbidity of 12-month DSM-IV disorders in the National Comorbidity Survey Replication. Archives of General Psychiatry 2005, 62:617-627.

3. Merikangas KR, He JP, Burstein M, Swanson SA, Avenevoli S, Cui L, Benjet C, Georgiades K, Swendsen J: Lifetime prevalence of mental disorders in U.S. adolescents: Results from the National Comorbidity Survey Replication-Adolescent Supplement (NCS-A). Journal of the American Academy of Child and Adolescent Psychiatry 2010, 49:980-989.

4. Budde M, Anderson-Schmidt H, Gade K, Reich-Erkelenz D, Adorjan K, Kalman JL, Senner F, Papiol S, Andlauer TFM, Comes AL, et al.: A longitudinal approach to biological psychiatric research: The PsyCourse study. American Journal of Medical Genetics Part B: Neuropsychiatric Genetics 2018.

5. Zanarini MC, Frankenburg FR, Hennen J, Reich DB, Silk KR: Axis I comorbidity in patients with borderline personality disorder: 6-year follow-up and prediction of time to remission. American Journal of Psychiatry 2004, 161:2108-2114. 
Future directions in personality pathology

6. Bateman AW, Gunderson J, Mulder R: Treatment of personality disorder. Lancet 2015, 385:735-743. [* This is the best and briefest summary of the current state of the art. Although now 5 years old, not a lot has changed. There are more recent summaries of the evidence but this paper states clearly what the evidence current and future means for personality disorder diagnosis.]

7. Goldberg D: Psychopathology and classification in psychiatry. Social Psychiatry and Psychiatric Epidemiology 2015, 50:1-5.

8. Zimmerman M, Morgan TA, Stanton K: The severity of psychiatric disorders. World Psychiatry 2018, 17:258-275. 8. Zimmerman M, Morgan TA, Stanton K: The severity of psychiatric disorders. World Psychiatry 2018, 17:258-275. [* This paper asks an unusual question of its readers: Do we know what we meant when we say someone has a "severe" depression (or other disorder)? It reviews evidence that says: "Not really". There are concepts in psychiatry and psychology used every day that have less than profound empirical support but because the word "severe" is commonly used, it is generally believed that someone somewhere knows what it means.]

9. Caspi A, Moffitt TE: All for one and one for all: Mental disorders in one dimension. American Journal of Psychiatry 2018, 175:831-844. [** This is the most thoughtful review of the $\mathrm{p}$ factor literature, and the classic statement of the problem from the researchers who started work on the topic.]

10. Lahey BB, Applegate B, Hakes JK, Zald DH, Hariri AR, Rathouz PJ: Is there a general factor of prevalent psychopathology during adulthood? Journal of Abnormal Psychology 2012, 121:971-977. 
Future directions in personality pathology

11. Lahey BB, Krueger RF, Rathouz PJ, Waldman ID, Zald DH: Validity and utility of the general factor of psychopathology. World Psychiatry 2017, 16:142-144.

12. Smith GT, Atkinson EA, Davis HA, Riley EN, Oltmanns JR: The general factor of psychopathology. Annual Review of Clinical Psychology 2020, 16:75-98. [** This is the most comprehensive review of the $\mathrm{p}$ factor literature. It gives the reader an excellent overview of the field. It is an excellent starting point for reading around this complex subject.]

13. Patalay P, Fonagy P, Deighton J, Belsky J, Vostanis P, Wolpert M: A general psychopathology factor in early adolescence. British Journal of Psychiatry 2015, 207:15-22.

14. Sharp C, Wright AG, Fowler JC, Frueh BC, Allen JG, Oldham J, Clark LA: The structure of personality pathology: Both general ('g') and specific ('s') factors? Journal of Abnormal Psychology 2015, 124:387-398. [* This is perhaps one of the most original papers on personality pathology of the past decade. The authors show that BPD is part of a general personality disorder factor. It is also strongly aligned with the points argued for by Section III in DSM-5.]

15. Fonagy $\mathrm{P}$, Luyten $\mathrm{P}$, Allison $\mathrm{E}$, Campbell $\mathrm{C}$ : What we have changed our minds about: Part 2. Borderline personality disorder, epistemic trust and the developmental significance of social communication. Borderline Personality Disorder and Emotion Dysregulation 2017, 4:9.

16. Lahey BB, Zald DH, Perkins SF, Villalta-Gil V, Werts KB, Van Hulle CA, Rathouz PJ, Applegate B, Class QA, Poore HE, et al.: Measuring the hierarchical general 
Future directions in personality pathology

factor model of psychopathology in young adults. International Journal of Methods in Psychiatric Research 2018, 27.

17. Vine V, Byrd AL, Mohr H, Scott LN, Beeney JE, Stepp SD: The structure of psychopathology in a sample of clinically referred, emotionally dysregulated early adolescents. Journal of Abnormal Child Psychology 2020.

18. Watts AL, Poore HE, Waldman ID: Riskier tests of the validity of the bifactor model of psychopathology. Clinical Psychological Science 2019, 7:1285-1303.

19. Bornovalova MA, Choate AM, Fatimah H, Petersen KJ, Wiernik BM: Appropriate use of bifactor analysis in psychopathology research: Appreciating benefits and limitations. Biological Psychiatry 2020, 88:18-27.

20. Sellbom M, Tellegen A: Factor analysis in psychological assessment research: Common pitfalls and recommendations. Psychological Assessment 2019, 31:14281441.

21. Franic S, Dolan CV, Borsboom D, van Beijsterveldt CE, Boomsma DI: Three-and-ahalf-factor model? The genetic and environmental structure of the CBCL/6-18 internalizing grouping. Behavior Genetics 2014, 44:254-268.

22. Lahey BB, Van Hulle CA, Singh AL, Waldman ID, Rathouz PJ: Higher-order genetic and environmental structure of prevalent forms of child and adolescent psychopathology. Archives of General Psychiatry 2011, 68:181-189.

23. Pettersson E, Anckarsater H, Gillberg C, Lichtenstein P: Different neurodevelopmental symptoms have a common genetic etiology. Journal of Child Psychology and Psychiatry 2013, 54:1356-1365.

24. Pettersson E, Larsson H, Lichtenstein P: Common psychiatric disorders share the same genetic origin: A multivariate sibling study of the Swedish population. Molecular Psychiatry 2016, 21:717-721. 
Future directions in personality pathology

25. Harden KP, Engelhardt LE, Mann FD, Patterson MW, Grotzinger AD, Savicki SL, Thibodeaux ML, Freis SM, Tackett JL, Church JA, et al.: Genetic associations between executive functions and a general factor of psychopathology. Journal of the American Academy of Child and Adolescent Psychiatry 2019.

26. Rosenstrom T, Czajkowski NO, Ystrom E, Krueger RF, Aggen SH, Gillespie NA, Eilertsen E, Reichborn-Kjennerud T, Torvik FA: Genetically informative mediation modeling applied to stressors and personality-disorder traits in etiology of alcohol use disorder. Behavior Genetics 2019, 49:11-23.

27. Song J, Bergen SE, Kuja-Halkola R, Larsson H, Landen M, Lichtenstein P: Bipolar disorder and its relation to major psychiatric disorders: A family-based study in the Swedish population. Bipolar Disorders 2015, 17:184-193.

28. Cross-Disorder Group of the Psychiatric Genomics Consortium: Identification of risk loci with shared effects on five major psychiatric disorders: a genome-wide analysis. Lancet 2013, 381:1371-1379.

29. Lee SH, Ripke S, Neale BM, Faraone SV, Purcell SM, Perlis RH, Mowry BJ, Thapar A, Goddard ME, Witte JS, et al.: Genetic relationship between five psychiatric disorders estimated from genome-wide SNPs. Nature Genetics 2013, 45:984-994.

30. Brainstorm Consortium, Anttila V, Bulik-Sullivan B, Finucane HK, Walters RK, Bras J, Duncan L, Escott-Price V, Falcone GJ, Gormley P, et al.: Analysis of shared heritability in common disorders of the brain. Science 2018, 360.

31. Macdonald AN, Goines KB, Novacek DM, Walker EF: Prefrontal mechanisms of comorbidity from a transdiagnostic and ontogenic perspective. Development and Psychopathology 2016, 28:1147-1175. [* An excellent review trying to understand the underpinnings of general psychopathology - this time in terms of mechanisms in the prefrontal cortex.] 
Future directions in personality pathology

32. Wise T, Radua J, Via E, Cardoner N, Abe O, Adams TM, Amico F, Cheng Y, Cole JH, de Azevedo Marques Perico C, et al.: Common and distinct patterns of greymatter volume alteration in major depression and bipolar disorder: evidence from voxel-based meta-analysis. Molecular Psychiatry 2017, 22:1455-1463.

33. Moberget T, Alnaes D, Kaufmann T, Doan NT, Cordova-Palomera A, Norbom LB, Rokicki J, van der Meer D, Andreassen OA, Westlye LT: Cerebellar gray matter volume is associated with cognitive function and psychopathology in adolescence. Biological Psychiatry 2019, 86:65-75.

34. Romer AL, Knodt AR, Houts R, Brigidi BD, Moffitt TE, Caspi A, Hariri AR: Structural alterations within cerebellar circuitry are associated with general liability for common mental disorders. Molecular Psychiatry 2018, 23:1084-1090.

35. Hinton KE, Lahey BB, Villalta-Gil V, Meyer FAC, Burgess LL, Chodes LK, Applegate B, Van Hulle CA, Landman BA, Zald DH: White matter microstructure correlates of general and specific second-order factors of psychopathology. NeuroImage. Clinical 2019, 22:101705.

36. Riem MME, van Hoof MJ, Garrett AS, Rombouts S, van der Wee NJA, van IMH, Vermeiren R: General psychopathology factor and unresolved-disorganized attachment uniquely correlated to white matter integrity using diffusion tensor imaging. Behavioural Brain Research 2019, 359:1-8. [* A fascinating report that is worth reading because it connects a range of discourses in relation to the $\mathrm{p}$ factor.]

37. Caspi A, Houts RM, Belsky DW, Goldman-Mellor SJ, Harrington H, Israel S, Meier MH, Ramrakha S, Shalev I, Poulton R, et al.: The p factor: One general psychopathology factor in the structure of psychiatric disorders? Clinical Psychological Science 2014, 2:119-137. 
Future directions in personality pathology

38. Beauchaine TP, Cicchetti D: Emotion dysregulation and emerging psychopathology: A transdiagnostic, transdisciplinary perspective. Development and Psychopathology 2019, 31:799-804. [* This paper introduces a special issue on emotion dysregulation. The issue contains the best summaries of this complex but fascinating field currently available.]

39. Campbell-Sills L, Barlow DH: Incorporating emotion regulation into conceptualizations and treatments of anxiety and mood disorders. In Handbook of emotion regulation. Edited by Gross JJ: Guilford Press; 2007:542-559.

40. Berking M, Wupperman P, Reichardt A, Pejic T, Dippel A, Znoj H: Emotion-regulation skills as a treatment target in psychotherapy. Behaviour Research and Therapy 2008, 46:1230-1237.

41. Gratz KL, Tull MT: Emotion regulation as a mechanism of change in acceptance-and mindfulness-based treatments. In Assessing mindfulness and acceptance processes in clients: Illuminating the theory and practice of change. Edited by Baer RA: New Harbinger Publications; 2010:107-133.

42. Greenberg LS: Emotion-focused therapy of depression. Person-Centered and Experiential Psychotherapies 2017, 16:106-117.

43. Lynch TR, Trost WT, Salsman N, Linehan MM: Dialectical behavior therapy for borderline personality disorder. Annual Review of Clinical Psychology 2007, 3:181-205.

44. Linehan MM: Cognitive-behavioral treatment of borderline personality disorder. New York, NY: Guilford Press; 1993.

45. Hayes SC: The act in context: The canonical papers of Steven C. Hayes. New York, NY: Routledge; 2015. 
Future directions in personality pathology

46. Chambers R, Gullone E, Allen NB: Mindful emotion regulation: An integrative review. Clinical Psychology Review 2009, 29:560-572.

47. Ellard KK, Fairholme CP, Boisseau CL, Farchione TJ, Barlow DH: Unified protocol for the transdiagnostic treatment of emotional disorders: Protocol development and initial outcome data. Cognitive and Behavioral Practice 2010, 17:88-101.

48. Sakiris N, Berle D: A systematic review and meta-analysis of the Unified Protocol as a transdiagnostic emotion regulation based intervention. Clinical Psychology Review 2019, 72:101751.

49. Sloan E, Hall K, Moulding R, Bryce S, Mildred H, Staiger PK: Emotion regulation as a transdiagnostic treatment construct across anxiety, depression, substance, eating and borderline personality disorders: A systematic review. Clinical Psychology Review 2017, 57:141-163.

50. Csibra G, Gergely G: Natural pedagogy as evolutionary adaptation. Philosophical Transactions of the Royal Society of London. Series B, Biological Sciences 2011, 366:1149-1157.

51. Tomasello M, Call J: Primate cognition. Oxford, UK: Oxford University Press; 1997.

52. Tomasello M: A natural history of human thinking. Cambridge, MA: Harvard University Press; 2014.

53. Russell BB: An inquiry into meaning and truth. London, UK: Allen \& Unwin; $1940 / 1967$.

54. Sperber D, Wilson D: Relevance: Communication and cognition edn 2nd. Malden, MA: Blackwell; 1995.

55. Csibra G: Recognizing communicative intentions in infancy. Mind \& Language 2010, 25:141-168. 
Future directions in personality pathology

56. Vouloumanos A, Waxman SR: Listen up! Speech is for thinking during infancy. Trends in Cognitive Sciences 2014, 18:642-646.

57. Egyed K, Király I, Gergely G: Communicating shared knowledge without language in infancy. Psychological Science 2013, 24:1348-1353. [* This is an exceptionally imaginative theory-driven study of the way social communication moderates the impact of learning experience.]

58. Futo J, Teglas E, Csibra G, Gergely G: Communicative function demonstration induces kind-based artifact representation in preverbal infants. Cognition 2010, 117:1-8.

59. Sperber D, Clement F, Heintz C, Mascaro O, Mercier H, Origgi G, Wilson D: Epistemic vigilance. Mind \& Language 2010, 25:359-393.

60. Recanati F: Can we believe what we do not understand? Mind \& Language 1997, 12:84-100.

61. Wilson D, Sperber D: Meaning and relevance. Cambridge, UK: Cambridge University Press; 2012.

62. Vygotsky LS: Mind in society: The development of higher psychological processes.

Edited by John-Steiner V, Cole M, Souberman E, Scribner S. Cambridge, MA: Harvard University Press; 1978.

63. Fonagy P, Luyten P, Allison E: Epistemic petrification and the restoration of epistemic trust: A new conceptualization of borderline personality disorder and its psychosocial treatment. Journal of Personality Disorders 2015, 29:575-609. 
Future directions in personality pathology

64. Fonagy P, Luyten P, Allison E, Campbell C: Reconciling psychoanalytic ideas with attachment theory. In Handbook of attachment, edn 3rd. Edited by Shaver P, Cassidy J: Guilford Press; 2016:780-804.

65. Fonagy $\mathrm{P}$, Luyten $\mathrm{P}$, Allison $\mathrm{E}$, Campbell $\mathrm{C}$ : What we have changed our minds about: Part 1. Borderline personality disorder as a limitation of resilience. Borderline Personality Disorder and Emotion Dysregulation 2017, 4:11.

66. Camras LA, Ribordy S, Hill J, Martino S, Spaccarelli S, Stefani R: Recognition and posing of emotional expressions by abused children and their mothers. Developmental Psychology 1988, 24:776-781.

67. Pollak SD, Cicchetti D, Hornung K, Reed A: Recognizing emotion in faces: Developmental effects of child abuse and neglect. Developmental Psychology 2000, 36:679-688.

68. Robin M, Pham-Scottez A, Curt F, Dugre-Le Bigre C, Speranza M, Sapinho D, Corcos M, Berthoz S, Kedia G: Decreased sensitivity to facial emotions in adolescents with borderline personality disorder. Psychiatry Research 2012, 200:417-421.

69. Luyten P, Campbell C, Allison E, Fonagy P: The mentalizing approach to psychopathology: State of the art and future directions. Annual Review of Clinical Psychology 2020, 16:297-325. [** This is a comprehensive review of the literature on mentalizing. It summarizes the progress in this field over the past two decades and aligns the therapeutic advances of mentalization-based treatment with the current trend for finding common elements in the processes underpinning psychological treatments.]

70. Borelli JL, Cohen C, Pettit C, Normandin L, Target M, Fonagy P, Ensink K: Maternal and child sexual abuse history: An intergenerational exploration of children's 
Future directions in personality pathology

adjustment and maternal trauma-reflective functioning. Frontiers in Psychology 2019, 10:1062.

71. Luyten P, Fonagy P: Mentalizing and trauma. In Handbook of mentalizing in mental health practice, edn 2nd. Edited by Bateman A, Fonagy P: American Psychiatric Publishing; 2019:79-99.

72. Ensink K, Begin M, Normandin L, Godbout N, Fonagy P: Mentalization and dissociation in the context of trauma: Implications for child psychopathology. Journal of Trauma \& Dissociation 2017, 18:11-30.

73. Berthelot N, Ensink K, Bernazzani O, Normandin L, Luyten P, Fonagy P:

Intergenerational transmission of attachment in abused and neglected mothers: The role of trauma-specific reflective functioning. Infant Mental Health Journal 2015, 36:200-212.

74. Lassri D, Luyten P, Fonagy P, Shahar G: Undetected scars? Self-criticism, attachment, and romantic relationships among otherwise well-functioning childhood sexual abuse survivors. Psychological Trauma: Theory, Research, Practice and Policy 2018, 10:121-129.

75. Christakis NA, Fowler JH: Social contagion theory: Examining dynamic social networks and human behavior. Statistics in Medicine 2013, 32:556-577.

76. Green B, Horel T, Papachristos AV: Modeling contagion through social networks to explain and predict gunshot violence in Chicago, 2006 to 2014. JAMA Internal Medicine 2017, 177:326-333.

77. Masuda N, Kurahashi I, Onari H: Suicide ideation of individuals in online social networks. PLOS ONE 2013, 8:e62262. 
Future directions in personality pathology

78. Rosenquist JN, Murabito J, Fowler JH, Christakis NA: The spread of alcohol consumption behavior in a large social network. Annals of Internal Medicine 2010, 152:426-433, W141.

79. Valente TW: Network interventions. Science 2012, 337:49-53.

\section{Highlights}

- A singular model for psychopathology will inform future directions for conceptualizing personality pathology.

- Understanding the development of and improving treatments for personality disorder will benefit from stronger integration of the impact of the wider social environment on psychopathology.

- Recent research on cultural transmission and social communication and learning can inform a more integrated approach to the development and treatment of personality disorder. 